\title{
Anyon braiding on a fractal lattice with a local Hamiltonian
}

\author{
Sourav Manna, ${ }^{1,2}$ Callum W. Duncan $\odot,{ }^{1,3}$ Carrie A. Weidner, ${ }^{4}$ Jacob F. Sherson, ${ }^{4}$ and Anne E. B. Nielsen ${ }^{1,4}$ \\ ${ }^{1}$ Max-Planck-Institut für Physik Komplexer Systeme, D-01187 Dresden, Germany \\ ${ }^{2}$ Department of Condensed Matter Physics, Weizmann Institute of Science, Rehovot 7610001, Israel \\ ${ }^{3}$ Department of Physics, SUPA and University of Strathclyde, Glasgow G4 ONG, United Kingdom \\ ${ }^{4}$ Department of Physics and Astronomy, Aarhus University, DK-8000 Aarhus C, Denmark
}

(Received 24 August 2021; accepted 31 January 2022; published 18 February 2022)

\begin{abstract}
There is a growing interest in searching for topology in fractal dimensions with the aim of finding different properties and advantages compared to the integer dimensional case. Here we construct a local Hamiltonian on a fractal lattice whose ground state exhibits topological braiding properties. The fractal lattice is obtained from a second-generation Sierpinski carpet with Hausdorff dimension 1.89. We use local potentials to trap and exchange anyons in the model, and the numerically obtained results for the exchange statistics of the anyons are close to the ideal statistics for quasiholes in a bosonic Laughlin state at half filling. For the considered system size, the energy gap is about three times larger for the fractal lattice than for a two-dimensional square lattice, and we find that the braiding results obtained on the fractal lattice are more robust against disorder. We propose a scheme to implement both fractal lattices and our proposed local Hamiltonian with ultracold atoms in optical lattices.
\end{abstract}

DOI: 10.1103/PhysRevA.105.L021302

Topologically ordered quantum systems harbor fractionalized excitations that are neither fermions nor bosons, but anyons [1,2]. Phases hosting anyons have been realized experimentally in solid-state systems in strong magnetic fields displaying the fractional quantum Hall effect [3-6]. Fractional quantum Hall phases also exist in systems defined on two-dimensional lattices, where the physical magnetic field is replaced by an artificial magnetic field, which can be much stronger [7-11]. Due to their unique degree of tunability, realizing fractional quantum Hall physics with ultracold atoms in optical lattices would give unique possibilities for investigating the effect in great detail, and there are currently several efforts towards achieving this for systems with few atoms [12-16]. The key components of artificial magnetic fields and topological band structures have already been prepared in several experiments [17].

Topological phases are mainly studied in systems with spatial (and Hausdorff) dimension one, two, and three, but recently interest has grown in studying topological models on fractal lattices with noninteger Hausdorff dimension. The Hausdorff dimension is a generalization of the dimension of a vector space and can provide a measure of how the details of a system change at different scales. While much of the knowledge generated in condensed-matter physics relies on the presence of an underlying Bravais lattice, fractal lattices do not fit into this framework and can hence give rise to different

Published by the American Physical Society under the terms of the Creative Commons Attribution 4.0 International license. Further distribution of this work must maintain attribution to the author(s) and the published article's title, journal citation, and DOI. Open access publication funded by the Max Planck Society. physics. Most of the studies of topological quantum models on fractal lattices so far have considered noninteracting systems [18-21], and those have, indeed, revealed new and interesting properties, including modifications of the Hofstadter butterfly and the presence of inner edge states. Much less is currently known about how fractal lattices affect the properties of topologically ordered phases of interacting systems. Initial steps have been taken by constructing Laughlin and Moore-Read trial states on fractal lattices [22,23], but the derived parent Hamiltonians of these states are nonlocal and involve many different types of interactions, making them difficult to realize. The study of models on fractal lattices is also motivated by experimental developments, such as the preparation of fractal models in molecules on surfaces [24,25]. It is desirable to realize fractal models of matter with ultracold atoms due to their ability to reach the regime of strongly interacting quantum systems and achieve single-site resolution [26,27].

Here we show that a system with only nearest-neighbor complex hopping and hardcore interactions on a finitegeneration fractal lattice can give rise to anyonic braiding properties, and we propose a scheme to implement the Hamiltonian experimentally with ultracold atoms in optical lattices. We use local potentials to trap anyons in the model, and we study their braiding properties under adiabatic time evolution. We find that the considered system with relatively few sites and particles is already enough to get braiding statistics close to the ideal value for quasiholes in a bosonic Laughlin state at half filling and to produce interesting differences compared to a corresponding model on a two-dimensional lattice with the same number of sites and particles. In particular, the gap between the ground state and the first excited state is approximately three times larger for the considered model on the fractal lattice. We also observe that the phase acquired by the wave function due to braiding is more robust with respect 
(a)

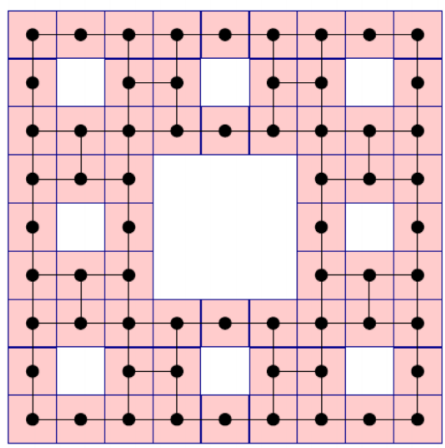

(b)

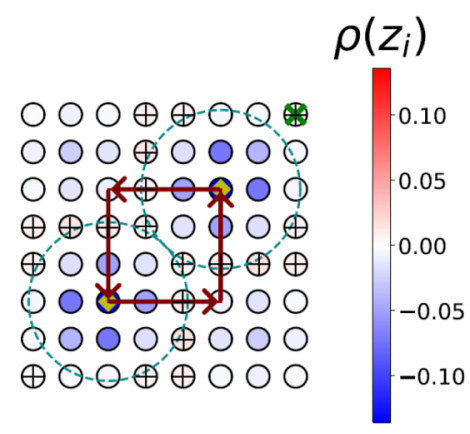

(c)

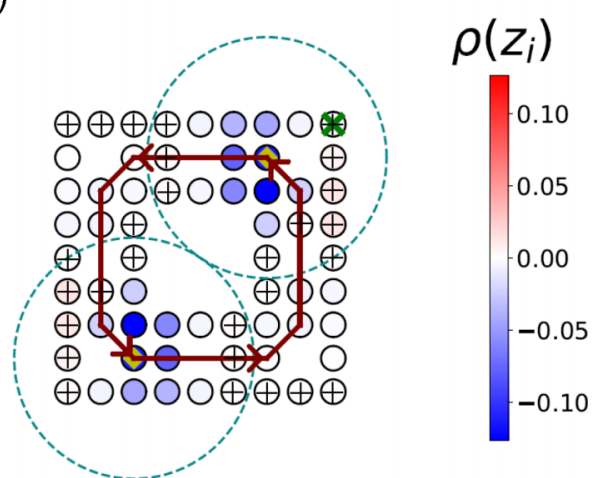

FIG. 1. (a) The model is defined on a second-generation Sierpinski carpet (red squares). The lattice sites are marked by circles, and the bonds connecting the sites illustrate the hopping terms. A magnetic flux goes through each lattice site in the direction perpendicular to the plane. (b, c) Anyons trapped on the square and fractal lattices. The anyon density profiles $\rho\left(z_{i}\right)$ from (5) when the anyon trapping potentials are on the sites marked by diamonds are shown with colors (gray scale). The sites with $\rho\left(z_{i}\right)>0$ are additionally marked with plus signs, while the sites with $\rho\left(z_{i}\right) \leqslant 0$ are not marked. It is seen that the anyons are screened. The dashed lines encircle the regions that we sum over when we compute the anyon charges. The arrows show the considered braiding path. We compute the Aharonov-Bohm phase by placing one of the anyons on the site marked by a cross while the other anyon follows the marked path.

to disorder on the fractal lattice, and the anyons are better screened. This robustness could be advantageous in future applications that utilize anyons, including the experimental proposal considered here, and the relatively small system size is also an advantage for implementations in ultracold atoms.

The proposed protocol to implement the Hamiltonian involves single-site addressing [28] and laser-assisted hopping tuned to achieve the desired phase factors [29]. We expect that the setup could also be used to generate integer quantum Hall phases on fractal lattices. The main challenge in realizing fractional quantum Hall phases in ultracold atoms in optical lattices is to reach the ground state, and the larger gap for the fractal lattice is hence an advantage. Generating optical, fractal lattices as described below also opens the door for studying various phenomena of quantum systems on fractal lattices.

Model. Typical ingredients required to obtain fractional quantum Hall physics include interactions and a magnetic field perpendicular to the plane. In lattice systems the magnetic field is often translated into corresponding complex hopping terms through the Peierls substitution [30], as we shall also do below. We start from a second-generation Sierpinski carpet [Fig. 1(a)] and obtain the considered fractal lattice by putting a lattice site in the center of each of the small squares. We denote the positions of the $N=64$ lattice sites in the complex plane by $z_{j}$ with $j \in\{1, \ldots, N\}$ and consider $M=4$ bosons on the lattice. The Hamiltonian

$$
H=-J \sum_{\langle j k\rangle} c_{j}^{\dagger} c_{k} e^{i \phi_{j k}}+U \sum_{l} n_{l}\left(n_{l}-1\right), \quad U \gg J,
$$

consists of complex, nearest-neighbor hopping terms of strength $J$ and an on-site interaction term of strength $U$. The operator $c_{k}$ annihilates a boson on the $k$ th lattice site, $n_{k}=$ $c_{k}^{\dagger} c_{k}$, and $\phi_{j k}$ is the phase the wave function acquires when a particle hops from $z_{k}$ to $z_{j}$. In the computations below, which are all done using exact diagonalization, we assume that $U / J$ is so large that one can neglect the possibility to have more than one boson on a site, i.e., we work with hardcore bosons.
The particular form of $\phi_{j k}$ is determined from the chosen magnetic field. In two-dimensional fractional quantum Hall models, the magnetic field is often either uniform or only penetrates the lattice sites. For a fractal lattice, it is similarly natural to let the magnetic field only penetrate the lattice sites, since then the pattern of magnetic flux also forms a fractal. We hence choose the magnetic field to be $\vec{B}(z)=\alpha \sum_{l} \delta\left(z-z_{l}\right) \hat{z}$, where $\alpha$ is the flux penetrating one lattice site measured in terms of the magnetic flux unit, $\delta$ is the Dirac $\delta$ function, and $\hat{z}$ is a unit vector perpendicular to the plane. This field configuration gives rise to the vector potential

$$
\vec{A}(z)=\sum_{l} \frac{\alpha \hat{\theta}_{l}}{\left|z-z_{l}\right|},
$$

where $\hat{\theta}_{l}$ is a unit vector in the plane rotated by $\pi / 2$ compared to $z-z_{l}$. From this we obtain

$$
\phi_{j k}=\int_{z_{k}}^{z_{j}} \vec{A}(r) \cdot \vec{d} l=\alpha \sum_{l(\neq j \neq k)} \operatorname{Im}\left[\ln \left(\frac{z_{j}-z_{l}}{z_{k}-z_{l}}\right)\right],
$$

where $\vec{d} l$ is an infinitesimal vector along the hopping direction. Below we take $M /(\alpha N)=1 / 2$, where $M$ is the number of particles. If the system is topological, we hence expect it to be in a bosonic Laughlin phase with quasiholes of charge $1 / 2$.

The model described above can also be defined on a square lattice, which we will do for comparison. We will consider the $8 \times 8$ quadratic lattice to allow for a proper comparison to the fractal lattice. We will consider open boundary conditions for both the square and fractal lattice for appropriate comparison. Note, periodic boundary conditions are not consistent with the fractal retaining its scaling nature.

Energy gap. The energy gap, $\delta E$, between the ground state and the first excited state is an important property of the model. This is due to the gap's relation to the state's stability, both in terms of robustness to disorder and feasibility of experimental implementations. We find that the size of the energy gap varies substantially with the number of particles and is particularly large for the fractal lattice with four particles. 
For four particles we find that the energy gap is about three times larger for the fractal lattice than for the square lattice. Specifically, the gap is $\delta E=0.313 \mathrm{~J}$ for the fractal lattice and $\delta E=0.105 \mathrm{~J}$ for the square lattice.

Creation of anyons. Quasiholes give rise to local reductions of the particle density, and therefore local potentials tend to trap them [31]. To obtain anyons in our model, we hence add local trapping potentials of the form

$$
H_{V}=V n_{l}+V n_{m}, \quad l \neq m, \quad U \gg V \gg J,
$$

to the Hamiltonian $H$ and simultaneously remove one particle. If the model is in a topological phase, we expect the potentials to trap one anyon at site $l$ and one anyon at site $m$. The anyons have a finite spread and hence also modify the densities on nearby sites. To show that anyons are indeed formed, we compute the charge and statistics of the anyons.

The anyon density profile,

$$
\rho\left(z_{i}\right)=\left\langle n_{i}\right\rangle_{H+H_{V}, M-1}-\left\langle n_{i}\right\rangle_{H, M},
$$

is the difference between the particle density for the ground state of $H+H_{V}$ with $M-1$ particles and the particle density for the ground state of $H$ with $M$ particles. We sum this quantity over a local region $\sigma_{k}$ around the position of the $k$ th potential to obtain the change in the number of particles within $\sigma_{k}$. The region should be large enough to enclose the complete anyon, and here we take $\sigma_{k}$ to be the sites inside the dashed circles in Figs. 1(b) and 1(c). Taking the charge of a particle to be -1 , the anyon charges

$$
Q_{k}=-\sum_{z_{i} \in \sigma_{k}} \rho\left(z_{i}\right), \quad k \in\{1,2\},
$$

evaluate to 0.466 for the square lattice and 0.480 for the fractal lattice, which are close to the expected value $1 / 2$. Note that $Q_{1}=Q_{2}$ in both cases due to symmetry. That the charges are close to $1 / 2$ shows that there is some screening in the system, but this can happen also in the absence of topology. We therefore now turn to computing the exchange statistics.

Fractional statistics. We now calculate the anyon exchange statistics to further characterize the type of anyons present in the system. We do this by adiabatically exchanging two anyons in the counterclockwise direction. This exchange results in the ground state $|\Psi\rangle$ of the Hamiltonian $H+H_{V}$ acquiring a Berry phase $\exp (i \pi \theta)$, defined by

$$
\theta=i \oint_{\mathcal{C}}\left\langle\Psi\left|\nabla_{w}\right| \Psi\right\rangle d w+\text { c.c. }
$$

where $w$ parametrizes the exchange path $\mathcal{C}$. There are two contributions to consider in $\theta$ : the Aharonov-Bohm phase $\theta_{\mathrm{AB}}$, since anyons circulate around the magnetic fluxes, and the statistical phase $\theta_{\mathrm{s}}$ of the anyons themselves. Therefore we have $\theta=\theta_{\mathrm{AB}}+\theta_{\mathrm{s}}$. The value of $\theta_{\mathrm{AB}}$ is obtained by circulating one anyon and keeping the other anyon fixed at a position sufficiently outside the moving anyon's path. The particular value of $\theta_{\mathrm{s}}(\neq[0,1])$ characterizes the type of anyons present in a given topological order.

To adiabatically move a trapping potential from the site $l$ to the nearby site $l^{\prime}$, we follow the procedure in Ref. [31] and consider the Hamiltonian

$$
H_{T}=H+(1-\gamma) V n_{l}+\gamma V n_{l^{\prime}}+V n_{m} .
$$

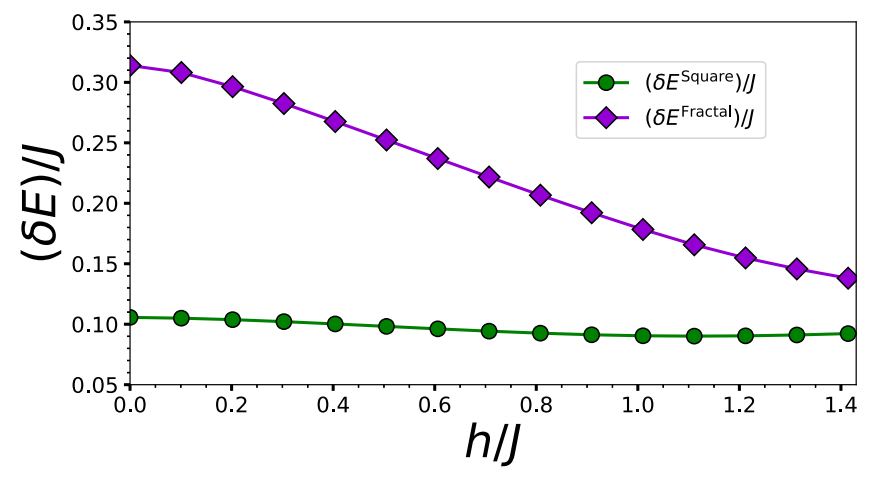

FIG. 2. The energy gap $\delta E / J$ between the ground state and the first excited state of the Hamiltonian (10) as a function of the disorder strength $h / J$ for the models on the square and fractal lattices with 64 sites and 4 particles. We average over 400 statistically independent disorder realizations for each $h$ to ensure convergence. The error bar is of order $10^{-3}$ for all data points. The energy gap is seen to be significantly larger for the model on the fractal lattice.

We vary $\gamma$ from 0 to 1 , following the ramp

$$
\gamma=\frac{\delta r}{r}-\frac{1}{2 \pi} \sin \left(\frac{2 \pi \delta r}{r}\right)
$$

with $r$ a number of steps sufficiently large to maintain adiabaticity and $\delta r \in[0,1, \ldots, r]$ as the individual step. We move one anyon at a time while keeping the other anyon fixed at its position to minimize overlap during the driving. The exact diagonalization used to obtain the ground state in each step does not fix the global phase factor of the state. We hence need to fix the global phase factor relative to the state at the beginning of the adiabatic evolution. We do this by choosing the global phase factor of the ground state in a given step such that its overlap with the ground state at the previous step is real.

We choose the exchange path for the square and fractal lattices shown in Fig. 1. The statistical phase of the anyons is found to be $\theta_{\mathrm{s}}=0.4589$ on the square lattice and $\theta_{\mathrm{s}}=$ 0.5089 on the Sierpinski carpet fractal lattice. These numbers are close to the expected value $\theta_{\mathrm{s}}=1 / 2$ for quasiholes in a bosonic Laughlin state at half filling. Therefore we conclude that the local Hamiltonian proposed here hosts anyons displaying Laughlin-type braiding statistics. We also note that if we instead choose a uniform magnetic field on the fractal lattice, we do not obtain a statistical phase close to $1 / 2$.

Effect of disorder. We next study the robustness of the braiding properties of the models with respect to weak disorder. We add a disordered potential at each lattice site and write the Hamiltonian as

$$
H^{\prime}=H+\sum_{i} h_{i} n_{i},
$$

where $h_{i} \in[-h, h]$ is drawn from a uniform random distribution with $h$ the disorder strength.

We plot in Fig. 2 the energy gap $\delta E / J$, averaged over random disorder realizations, between the ground state and the first excited state as a function of $h / J$ for both the models 
TABLE I. Exchange phases for a single disorder realization on the square and fractal lattices. Overlap between the charge distributions of the two anyons leads to deviations from the ideal value $\theta_{\mathrm{s}}=1 / 2$. The two numbers provided for disorder strength $h / J=1$ correspond to two different disorder realizations.

\begin{tabular}{lcc}
\hline \hline Disorder & $\theta_{\text {s }}$ on square lattice & $\theta_{\text {s }}$ on fractal lattice \\
\hline$h / J=0$ & 0.4589 & 0.5089 \\
$h / J=0.25$ & 0.0570 & 0.4803 \\
$h / J=0.5$ & 0.2615 & 0.5034 \\
$h / J=1$ & $0.0977 ; 0.0994$ & $0.5171 ; 0.5479$ \\
\hline \hline
\end{tabular}

on the square lattice and on the fractal lattice with 64 sites and four particles. The model acquires a larger gap on the fractal lattice than that on the square lattice, which could be significant for its realization. This gap reduces with the introduction of disorder but is still substantially larger than that of the square lattice over large ranges of disorder.

Evaluating the braiding statistics of the anyons is a computationally expensive task. Therefore we consider the case of $h=0$ and three $h \neq 0$ values where we take a single or two disorder realizations. We find that the statistics of the anyons are approximately $\theta_{\mathrm{s}}=1 / 2$ on the fractal lattice for the considered disorder strengths, see Table I. For the square lattice, however, we find that the anyon statistics is destroyed already for $h / J=0.25$. Inspecting the anyon density profiles along the trajectory, we find that the anyons are not well separated at all times. Thus, for the square lattice we are hence not able to draw conclusions about the statistics without considering a larger lattice. In the fractal lattice the anyons are better screened, which allows for robust braiding in a smaller system.

Proposal for implementing the Hamiltonian. An experimental demonstration of the Sierpinski carpet fractal lattice in a cold-atom system requires two components: efficient preparation of the lattice system with the desired filling factor and generation of the required site-to-site hopping phases. For the former, we assume that we start by loading a single plane of a three-dimensional cubic lattice in a conventional quantum-gas microscopy system [26,27] capable of imaging atoms with single-site resolution using a high-numerical-aperture (NA) microscope objective. Using spin-addressing techniques, a set number of atoms can be loaded into the lattice [28].

We now discuss the problem of generating the desired site-to-site hopping magnitudes and phases via light-assisted tunneling [29,32]. In general, tunneling is inhibited in the system if there exists an energy gradient along $x$ and $y$ giving rise to a bias $\Delta$ between each adjacent site. Light-assisted tunneling between adjacent sites can be restored if a pair of running-wave beams is added to the system. Given that the frequency difference between the running waves satisfies the relation $\omega=\omega_{1}-\omega_{2}= \pm \Delta / \hbar$, atoms are again allowed to tunnel between adjacent sites. The two light fields need only be present where the Wannier functions overlap significantly (that is, between adjacent lattice sites) [33]. Therefore we can control the magnitude of the effective tunneling parameter through control of the amplitudes of the two running waves we project onto the system. In Refs. [29,32], the hopping phases were controlled via the relative directions of the two running waves. Here, however, we propose to control the amplitude and phase of the tunneling parameter by locally shaping one of these two running waves using a spatial light modulator (SLM) [34,35].

The required tunneling phases are controlled via projection of two counterpropagating light potentials from the top and bottom of the lattice, respectively, with both beams running orthogonal to the lattice axes. The first laser acts as a light sheet onto the atoms from one direction and does not require high-resolution capabilities. Then, through the highresolution objective, one can project a second light-based potential with a phase and amplitude pattern mapped onto it via a SLM [35]. In this way one can engineer the local tunneling properties by carefully configuring the system so that light is present only between the adjacent lattice sites where tunneling is desired. If the resolution of the objective is high enough such that the point-spread function of the projection system is comparable to (or smaller than) the distance between lattice sites (see, e.g., the system in Ref. [36]), one can project these light potentials onto the lattice with minimal crosstalk between sites. Even in the presence of small amounts of crosstalk, the SLM-generated light field can readily be modified such that the desired field amplitudes and phases are generated at each lattice site. Finally, given that crosstalk is small, SLMs can also be used to project (again through the high-resolution objective) the local trapping potentials required for anyon generation and exchange.

Conclusions. We have constructed a local Hamiltonian that shows topological braiding on both the Sierpinski carpet, which is itself a fractal lattice, and a square lattice. The proposed Hamiltonian is found to lead to a larger energy gap between the topological ground state and the first excited state on the fractal lattice with 64 sites and 4 particles than that on a square lattice of the same size. We have also found that the fractal lattice enhances the robustness of the braiding properties of the topological state against the effects of disorder. We hypothesize that this enhancement is due to the different noninteger scaling dimension that characterizes the fractal lattice, and future research in this direction is warranted. We have proposed an experimental implementation of the local model introduced here with ultracold atoms in optical lattices. The experimental realization of the local model studied allows for the consideration of different geometries of the lattice, including different fractal lattices that could support topological order.

Acknowledgments. The authors thank W. Wang for discussions on related topics. This work was supported by the Independent Research Fund Denmark under Grant No. 804900074B and by the Carlsberg Foundation through a Semper Ardens grant. Work at the University of Strathclyde was supported by the EPSRC Quantum Technologies Hub for Quantum Computing and Simulation (EP/T001062/1). S.M. thanks the Weizmann Institute of Science, Israel Deans Fellowship through the Feinberg Graduate School for financial support. 
[1] J. M. Leinaas and J. Myrheim, On the theory of identical particles, Nuovo Cim. B 37, 1 (1977).

[2] F. Wilczek, Quantum Mechanics of Fractional-Spin Particles, Phys. Rev. Lett. 49, 957 (1982).

[3] D. C. Tsui, H. L. Stormer, and A. C. Gossard, Two-Dimensional Magnetotransport in the Extreme Quantum Limit, Phys. Rev. Lett. 48, 1559 (1982).

[4] R. B. Laughlin, Anomalous Quantum Hall Effect: An Incompressible Quantum Fluid with Fractionally Charged Excitations, Phys. Rev. Lett. 50, 1395 (1983).

[5] D. Arovas, J. R. Schrieffer, and F. Wilczek, Fractional Statistics and the Quantum Hall Effect, Phys. Rev. Lett. 53, 722 (1984).

[6] K. I. Bolotin, F. Ghahari, M. D. Shulman, H. L. Stormer, and P. Kim, Observation of the fractional quantum Hall effect in graphene, Nature (London) 462, 196 (2009).

[7] A. S. Sørensen, E. Demler, and M. D. Lukin, Fractional Quantum Hall States of Atoms in Optical Lattices, Phys. Rev. Lett. 94, 086803 (2005).

[8] M. Hafezi, A. S. Sørensen, E. Demler, and M. D. Lukin, Fractional quantum Hall effect in optical lattices, Phys. Rev. A 76, 023613 (2007).

[9] E. Kapit and E. Mueller, Exact Parent Hamiltonian for the Quantum Hall States in a Lattice, Phys. Rev. Lett. 105, 215303 (2010).

[10] N. Regnault and B. A. Bernevig, Fractional Chern Insulator, Phys. Rev. X 1, 021014 (2011).

[11] T. Neupert, L. Santos, C. Chamon, and C. Mudry, Fractional Quantum Hall States at Zero Magnetic Field, Phys. Rev. Lett. 106, 236804 (2011).

[12] N. R. Cooper and J. Dalibard, Reaching Fractional Quantum Hall States with Optical Flux Lattices, Phys. Rev. Lett. 110, 185301 (2013).

[13] J. Motruk and F. Pollmann, Phase transitions and adiabatic preparation of a fractional Chern insulator in a boson cold-atom model, Phys. Rev. B 96, 165107 (2017).

[14] Y.-C. He, F. Grusdt, A. Kaufman, M. Greiner, and A. Vishwanath, Realizing and adiabatically preparing bosonic integer and fractional quantum Hall states in optical lattices, Phys. Rev. B 96, 201103(R) (2017).

[15] M. Raciunas, F. N. Ünal, E. Anisimovas, and A. Eckardt, Creating, probing, and manipulating fractionally charged excitations of fractional Chern insulators in optical lattices, Phys. Rev. A 98, 063621 (2018).

[16] C. Repellin, J. Léonard, and N. Goldman, Fractional Chern insulators of few bosons in a box: Hall plateaus from centerof-mass drifts and density profiles, Phys. Rev. A 102, 063316 (2020).

[17] N. R. Cooper, J. Dalibard, and I. B. Spielman, Topological bands for ultracold atoms, Rev. Mod. Phys. 91, 015005 (2019).

[18] M. Brzezińska, A. M. Cook, and T. Neupert, Topology in the Sierpiński-Hofstadter problem, Phys. Rev. B 98, 205116 (2018).

[19] M. Fremling, M. van Hooft, C. M. Smith, and L. Fritz, Existence of robust edge currents in Sierpiński fractals, Phys. Rev. Research 2, 013044 (2020).
[20] S. Pai and A. Prem, Topological states on fractal lattices, Phys. Rev. B 100, 155135 (2019).

[21] A. A. Iliasov, M. I. Katsnelson, and S. Yuan, Hall conductivity of a Sierpiński carpet, Phys. Rev. B 101, 045413 (2020).

[22] S. Manna, B. Pal, W. Wang, and A. E. B. Nielsen, Anyons and fractional quantum Hall effect in fractal dimensions, Phys. Rev. Research 2, 023401 (2020).

[23] S. Manna, N. S. Srivatsa, J. Wildeboer, and A. E. B. Nielsen, Quasiparticles as detector of topological quantum phase transitions, Phys. Rev. Research 2, 043443 (2020).

[24] J. Shang, Y. Wang, M. Chen, J. Dai, X. Zhou, J. Kuttner, G. Hilt, X. Shao, J. M. Gottfried, and K. Wu, Assembling molecular Sierpiński triangle fractals, Nat. Chem. 7, 389 (2015).

[25] S. Kempkes, M. Slot, S. Freeney, S. Zevenhuizen, D. Vanmaekelbergh, I. Swart, and C. M. Smith, Design and characterization of electrons in a fractal geometry, Nat. Phys. 15, 127 (2019).

[26] W. S. Bakr, J. I. Gillen, A. Peng, S. Fölling, and M. Greiner, A quantum gas microscope for detecting single atoms in a Hubbard-regime optical lattice, Nature (London) 462, 74 (2009).

[27] J. F. Sherson, C. Weitenberg, M. Endres, M. Cheneau, I. Bloch, and S. Kuhr, Single-atom-resolved fluorescence imaging of an atomic Mott insulator, Nature (London) 467, 68 (2010).

[28] C. Weitenberg, M. Endres, J. Sherson, M. Cheneau, P. Schauß, T. Fukuhara, I. Bloch, and S. Kuhr, Single-spin addressing in an atomic Mott insulator, Nature (London) 471, 319 (2011).

[29] M. Aidelsburger, M. Atala, M. Lohse, J. T. Barreiro, B. Paredes, and I. Bloch, Realization of the Hofstadter Hamiltonian with Ultracold Atoms in Optical Lattices, Phys. Rev. Lett. 111, 185301 (2013).

[30] R. Peierls, Zur Theorie des Diamagnetismus von Leitungselektronen, Zeitschrift für Physik 80, 763 (1933).

[31] E. Kapit, P. Ginsparg, and E. Mueller, Non-Abelian Braiding of Lattice Bosons, Phys. Rev. Lett. 108, 066802 (2012).

[32] M. E. Tai, A. Lukin, M. Rispoli, R. Schittko, T. Menke, D. Borgnia, P. M. Preiss, F. Grusdt, A. M. Kaufman, and M. Greiner, Microscopy of the interacting Harper-Hofstadter model in the two-body limit, Nature (London) 546, 519 (2017).

[33] M. Aidelsberger, S. Nascimbéne, and N. Goldman, Artificial gauge fields in materials and engineered systems, C. R. Phys. 19, 394 (2018).

[34] F. Nogrette, H. Labuhn, S. Ravets, D. Barredo, L. Béguin, A. Vernier, T. Lahaye, and A. Browaeys, Single-Atom Trapping in Holographic 2D Arrays of Microtraps with Arbitrary Geometries, Phys. Rev. X 4, 021034 (2014).

[35] P. Zupancic, P. Preiss, R. Ma, A. Lukin, M. Tai, M. Rispoli, R. Islam, and M. Greiner, Ultra-precise holographic beam shaping for microscopic quantum control, Opt. Express 24, 13881 (2016).

[36] C. Robens, S. Brakhane, W. Alt, F. Kleißler, D. Meschede, G. Moon, G. Ramola, and A. Alberti, High numerical aperture $(\mathrm{NA}=0.92)$ objective lens for imaging and addressing of cold atoms, Opt. Lett. 42, 1043 (2017). 